\title{
Scalable-detail modular models for simulation studies on energy efficiency
}

\author{
Marco Bonvini*, Alberto Leva \\ Dipartimento di Elettronica e Informazione, Politecnico di Milano \\ Via Ponzio 34/5, 20133 Milano, Italy \\ \{bonvini, leva\}@elet.polimi.it \\ ${ }^{*} \mathrm{PhD}$ student at the Dipartimento di Elettronica e Informazione
}

\begin{abstract}
Simulation is widely used to assess and/or improve the energy efficiency of both existing and new buildings. Such an analysis has to account for heterogeneous phenomena efficiently, to manage components in a modular manner, and (which is seldom addressed in a structured way) to scale the detail level in all or part of the model, based on the particular simulation goal. In this manuscript, a proposal is formulated on how to structure a Modelica library so as to satisfy such a need.

Keywords: Building simulation; energy optimisation; object-oriented modelling; modular modelling; scalable detail.
\end{abstract}

\section{Introduction}

At each step of the design or refurbishing of a building, decisions need taking, based on some goal and on the state of the project, i.e., the decisions taken in the past. Like any engineering process, building (re)design is in fact a cyclic activity, where any choice has to be reconsidered when its effects - no matter how later observed - are found to be unsatisfactory.

Most of the mentioned decisions are complex, however, and to gather the necessary information for them, simulation is often the only viable way to go. In an ideal world, a simulation model should thus be available on the engineer's desk throughout the project, ready to help for complex decisions like a pocket calculator helps for simple computations. It should be possible to simulate the project at any time, irrespective of what was already fully designed, and what conversely was only specified in terms of the boundary conditions provided for the rest of the overall system. It should also be possible to move back and forth among the complexity levels implicitly defined above, in the case some past decision needs re-discussing.
Moreover, as the project moves toward its maturity and the model becomes correspondingly complicated - one should still have the possibility of replacing parts of said model with simpler ones, so as to improve simulation speed when only some aspects of the building's behaviour need investigating. And of course, the effort required to create the simulation model and keep it aligned to the project must be reasonable, i.e., adequately compensated by design quality improvements.

Such an approach to simulation is very different from those adopted by typical engineering tools. Most are domain-specific (e.g., electrical, Energy System or ES, Computational Fluid Dynamics or CFD, and so forth), or have limited flexibility (e.g., there is a library of pre-built "boiler" models and creating a new one is very far from trivial), or do not allow for a structured management of the models and simulations within a project, or any combination thereof. Needless to say, adopting the Object-Oriented Modelling and Simulation (OOMS) paradigm, and in particular the Modelica language, is a very promising idea.

In fact, several Modelica libraries for building simulation already exist $[1,2,5]$. However, the use of such libraries as a decision aid along the evolution of a project still experiences some difficulties. This work presents the authors' opinion on the matter, and proposes a possible modus operandi to solve the encountered problems.

\section{Problem statement}

Traditionally, the (re)design of a building is treated as the partially disjoint (explanations follow) design of its "subsystems". Although there is no standardised nomenclature, in fact, virtually the totality of engineering tools broadly distinguish (a) the "building" stricto sensu or "containment", i.e., walls, doors, win- 
dows and so on, (b) the contained air volumes, possibly divided in zones, (c) the Heating, Ventilation and Air Conditioning (HVAC) system, (d) automation and control systems, and (e) energy sources/sinks owing to the building utilisation, e.g., the heat released by occupants, industrial machines, or whatever is installed. The subsystems' interaction is accounted for by having some of them provide boundary conditions for the design of some other.

This is apparently very far from a really integrated approach, whence the term "partially disjoint" applied above to current design practices, but tools that address the simulation of all (or at least part) of the subsystems in a coordinated way are at present little more than research objects $[3,5,4]$.

There is more than one reason for such a scenario. The most widely acknowledged one is given by the very different issues posed by the various subsystems. For example, control system models are made of oriented blocks and may need sometimes a continuoustime and sometimes a digital representation depending on the simulation purpose; models for HVAC, conversely, live invariantly in the continuous-time domain, but are typically zero- or one-dimensional, while models of phenomena that occur in continua such as a wall or an air volume often cannot avoid threedimensional spatial distributions.

However, at least another reason needs mentioning. During its design, a building is looked at by various professionals, each one considering one or a few subsystems, and adopting a specific schematisation, ranging from $2 \mathrm{D}$ or $3 \mathrm{D} \mathrm{CAD}$ drawings to piping diagrams, electrical schemes, and so forth. Apparently none of those schematisations is suitable for system-level modelling, which means that some new ones need introducing - whence a further difficulty.

Moreover, the designed diagrams tend to reach their final detail in a very few steps: for example a heating system may be specified as a P\&ID, but then it is typically drawn in its complete layout, and more or less same is true for structures, walls, shadings, and so on.

As any expert knows, the development and maintenance of a simulation model follows a completely different path, especially if the model is conceived as a design decision aid. It must not be necessary to know much building details before being able to perform the first simulation, contrary to what one may be led to think, based on how most Modelica libraries on this matter (including those developed by the authors, of course) are structured.

In synthesis, our opinion is that structuring a Mod- elica library for building simulation as a decision aid, is better done based on the detail levels one needs throughout a study. It should be stressed, for the sake of clarity, that we are dealing with the structuring of a library, not (necessarily) of models built from it. The aim is to facilitate the construction of said models in the most effective way to follow the project cycle. Of course, after such a structuring, most of the connector abstraction work will go on in the traditional way, but the aspect just mentioned remains the key one.

\section{A library structuring proposal}

As anticipated, simulation-based analysis needs conducting at different levels of detail. This remark can lead to a library structuring, which we propose to carry out in three steps.

\subsection{Step 1}

The first step is to define and qualify the mentioned detail levels. In this work we define four ones, corresponding to the basic questions encountered along a building project. Of course the matter is more articulated, and one could consider defining more levels, or further customising them based on the needs of some particular class of applications. For each defined level, we point out

- the purpose, i.e., what type of analysis it is conceived for;

- the hypotheses under which its models are valid;

- the analysis protocol, i.e., how the intended analysis is to be performed;

- the structural limitations, i.e., what facts the models are by construction unable to capture, and thus are implicitly considered neglectable in the intended analysis;

- the practice-based limitations, i.e., for example, what the models could in principle represent, but it is not convenient/cost-effective to have represented;

- and finally the (main) decision-making usefulness of the models.

\section{Level 0}

Purpose: determine/verify the overall first-cut energy needs on a static basis. 
Hypotheses: the (single) internal air temperature follows the prescribed, constant set point; thermal capacities are disregarded; external ambient conditions are fixed; air renovation and exogenous energy sources are fixed based on the assumed utilisation.

Analysis protocol: a (static) simulation is done for each relevant scenario (e.g. a best and a worst case are defined for each climatic period in a year) and then results are combined in a straightforward manner.

Structural limitations: no dynamic phenomenon (due e.g. to heat storage) is accounted for, the source of the required energy is not discussed, no cost model is correspondingly introduced.

Practice-based limitations: it is generally inconvenient to introduce at this stage detailed models of the building containment (e.g., shading devices), whence a further source of approximation.

Decision-making usefulness: first overall assessment of the energy needs; possibility of evaluating highlevel alternatives (e.g., it is already possible to roughly estimate the benefits of a certain type of insulation).

Note, incidentally, that level 0 is similar to that of (basic) energy certification analyses.

\section{Level 1}

Purpose: determine the overall energy needs accounting for internal thermal zones and heat storages in the containment.

Hypotheses: same as level 0 but with various internal air zones' temperatures, that follow the prescribed set points (here not constant) possibly filtered through some low-order dynamics to account for the control system's action, or at most with simplified descriptions of local controls; also, containment thermal capacities are considered.

Analysis protocol: same as level 0 except that here simulations are apparently dynamic.

Structural limitations: here too the source of the required energy is not considered (i.e., only the energy need is modelled, irrespective of the used mix of available sources), and no cost model is introduced.

Practice-based limitations: at this stage it can make sense to use detailed models of the building containment, while precise hypotheses on the control system's behaviour may be premature.

Decision-making usefulness: dynamic assessment of the energy needs, and possibility of evaluating highlevel alternatives also regarding energy storages (e.g. the slower thermal behaviour typically induced by insulation is evidenced, and the temperature set point profiles can be discussed accordingly).

\section{Level 2}

Purpose: size/design/assess the energy system (ES) and discuss the energy mix.

Hypotheses: same as level 1 but air zones' thermal capacities are considered and the zone-level control system is introduced, including a reasonably detailed description of its physical realisation.

Analysis protocol: same as level 1.

Structural limitations: here the energy sources come into play but no detailed model of the generating devices (e.g. boilers) is used yet.

Practice-based limitations: at this stage reasonably detailed models of both the building containment and the zone-level control system are advised, while hypotheses on the energy sources are still coarse.

Decision-making usefulness: dynamic assessment of the ES and the zone-level controls capability of fulfilling the energy needs, including the discussion of possible alternatives (e.g. for the control system structuring and the energy mix) assuming an ideal behaviour of the energy sources.

\section{Level 3}

Purpose: size/design/assess the energy sources and the integrated control system, possibly including costs Hypotheses: same as level 2 but more detailed models of the energy sources, and possibly the central controls, are introduced.

Analysis protocol: same as level 2.

Structural and practice-based limitations: conceptually this is the most detailed model possible with the available information, the only limitations come from errors in said information.

Decision-making usefulness: dynamic assessment of the integrated central and zone-level controls, possible optimisation of the set point curves based on cost considerations.

\subsection{Step 2}

The second step is to observe that the same detail levels above can be viewed from the model components' standpoint, resulting in the definition of which phenomena to represent, and how, in each of them. A synthetic list is given below.

\section{Level 0}

Containment elements: thermal conductances, possibly computed based on stratigraphies; correlations for solar radiation captation and exchanges with air/sky/terrain.

Internal air: a single prescribed temperature (scenario-based). 
External ambient condition and solar radiation: prescribed (scenario-based).

Air renovation: prescribed flow rates (scenariobased).

ES: absent.

Exogenous energy sources (e.g. from machines, inhabitants, and so forth): fixed powers (scenario-based).

Control system: absent.

\section{Level 1}

Containment elements: same as level 0 but thermal capacities are introduced.

Internal air: a prescribed temperature per zone, possibly dynamically filtered (scenario-based), or some very simple description of local controls (but not of their physical realisation).

External ambient condition and solar radiation: same as level 0.

Air renovation: same as level 0.

ES: absent.

Exogenous energy sources: prescribed powers variable in time (scenario-based).

Control system: de facto absent if its action is summarised in the set point filters' time constants, or extremely simplified, see above.

\section{Level 2}

Containment elements: same as level 1.

Internal air: thermal capacities (possibly Mollierbased descriptions if humidity needs considering).

External ambient condition and solar radiation: same as level 1.

Air renovation: governed by the control system.

$E S$ : piping and HVAC elements present, energy sources assumed to behave ideally (e.g. a boiler delivers the required flow rate at the required temperature). Exogenous energy sources: same as level 1.

Control system: zonal controls represented, central ones idealised (in accordance with the partial ES representation).

\section{Level 3}

Containment elements: same as level 2.

Internal air: same as level 2.

External ambient condition and solar radiation: same as level 2.

Air renovation: same as level 2.

$E S$ : same as level 2 but models for the energy sources are introduced.

Exogenous energy sources: same as level 2.

Control system: both central and zonal controls represented.

\subsection{Step 3}

The final step is to structure the library so that each component, preserving the physical interfaces, be described by different models depending on the required detail level. For example, in the following, wall or air models have the same connectors, but their equations change with the detail level, while the energy system model grows with said level, being firstly a mere impressed power, then piping and exchangers with prescribed water inlet conditions, then the complete circuit. Given the scope of this work, the matter is discussed in the next section, based on a representative example that synthetically covers all the detail levels.

\section{Application}

This section illustrates how, along the proposed approach, scalable-detail models are able to support a designer through the phases of a typical project. For simplicity, the addressed design refers to the temperature control of a single room. The room is $3 \times 3 \times 2.5 \mathrm{~m}$ in size, surrounded by walls of $0.4 \mathrm{~m}$ thickness. Concerning the walls, their thermal conductivity is 1.91 $W /(m K)$, their density is $2400 \mathrm{~kg} / \mathrm{m}^{3}$ and their thermal capacity is $880 \mathrm{~J} /(\mathrm{kgK})$. The convective heat transfer coefficient between the walls and the air of the room is $5 \mathrm{~W} /\left(\mathrm{m}^{2} K\right)$, while that between walls and the environment is $10 \mathrm{~W} /\left(\mathrm{m}^{2} \mathrm{~K}\right)$. The temperature of the environment that surrounds the room is kept constant at $10{ }^{\circ} \mathrm{C}$. The design objective is to maintain the air temperature in the room at $20^{\circ} \mathrm{C}$.

\subsection{Level 0: overall static energy needs as- sessment}

In this phase, the designer's question is "how much power is needed in order to maintain the room (or a building) at a certain temperature level, given the envelope transmittance and assigned environmental conditions?" The answer to this (level 0) question can be obtained by static models such as that of figure 1 .

At this level, transients are neglected, and heat flow rates are computed based only on thermal conduction and convection at steady state, when the temperature of the room has reached the desired value.

There is not the space here to enter into Modelica details. Suffice however to say that in figure (1) the air model (white cube) is a mere heat capacity, the wall models are multilayer thermal resistances plus an additional heat capacity, that when evaluated as parameter causes the Fourier-based heat transfer law to 


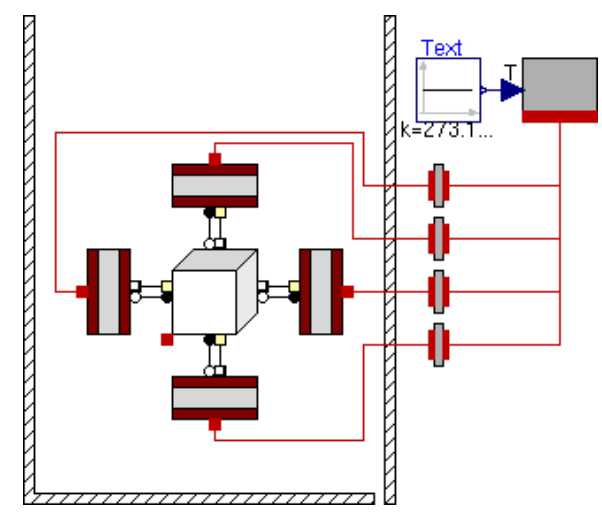

Figure 1: (Level 0) static analysis of the room only, considered as a mass of air at constant temperature $T=20^{\circ} \mathrm{C}$.

switch from dynamic to static in the case of zero capacity. Walls are connected to the air with two connectors: one simply carries the temperature as effort and the heat rate as flow variable, while the second conveys information about the air velocity for the subzonal room model mentioned later on: needless to say, such information is not used at the detail level of this section. The " $T$ " block on the upper right side simply prescribes the temperature on its temperature/heat rate connector.

\subsection{Level 1: dynamic energy needs assess- ment and local controls}

According to the static model of figure (1), the power needed to maintain this steady state condition is $647.79 \mathrm{~W}$. Scaling up the level of detail, this first result can be compared with a dynamic simulation.

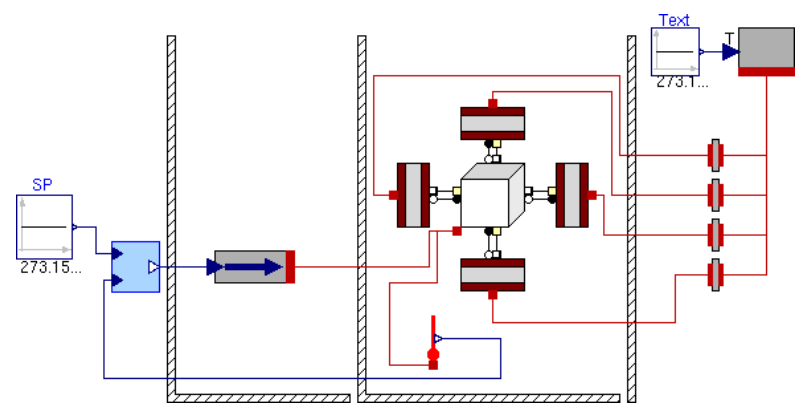

Figure 2: (Level 1) dynamic analysis of the room only, plus local controls. At this stage walls and air within the room are dynamic models. A simple control system, that directly injects power in the room, is introduced.

At this level, see figure (2), heat storages are considered, therefore heat capacity of walls and air are included. A very simple control system is also introduced at the local level, while the conditions of the heating water centrally supplied are still impressed. The presented analysis is therefore de facto a level 1 one, and local controls are represented: the case where controls are conversely idealised is here skipped for brevity. The Modelica elements of figure (2) are the same as those of figure (1), plus a block prescribing the heat rate on its connector (near the centre) and an antiwindup, continuous-time PI controller (on the left).

Figure (4) shows the temperature transient, while figure (5) reports the power supplied by the control system to the room in order to maintain the prescribed temperature. This analysis shows that at steady state the amount of power predicted by the static analysis was correct, and the peak of power asked to the heating system in order to satisfy a certain response is higher than the final value (about $745 \mathrm{~W}$ ). It is clear that this analysis is more complete than the previous one, because without considering dynamic effects (i.e., sizing the equipment based on information provided by static models only) the risk of incorrectly estimating the real needs is notoriously high.

\subsection{Level 2: the energy system is brought in}

At this point the question is "How does the energy (heating) system need to be sized and controlled in order to provide the required power to the system?" Such a question can be answered by further detailing the model as indicated before, but of the focus is set on the energy system exclusively, one could detail that system and at the same time scale down the level of complexity of the room, for example re-considering it as a mass of air at constant temperature (the worst case is when the temperature of the room has reached its maximum, i.e., the Set Point value of $20^{\circ} \mathrm{C}$ ).

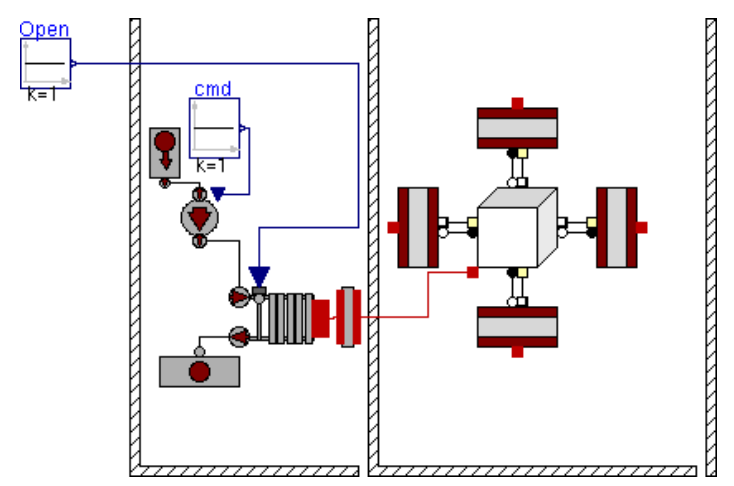

Figure 3: Level 2 (simplified) analysis of the heater only.

Figure (3) shows the new scheme. As anticipated 


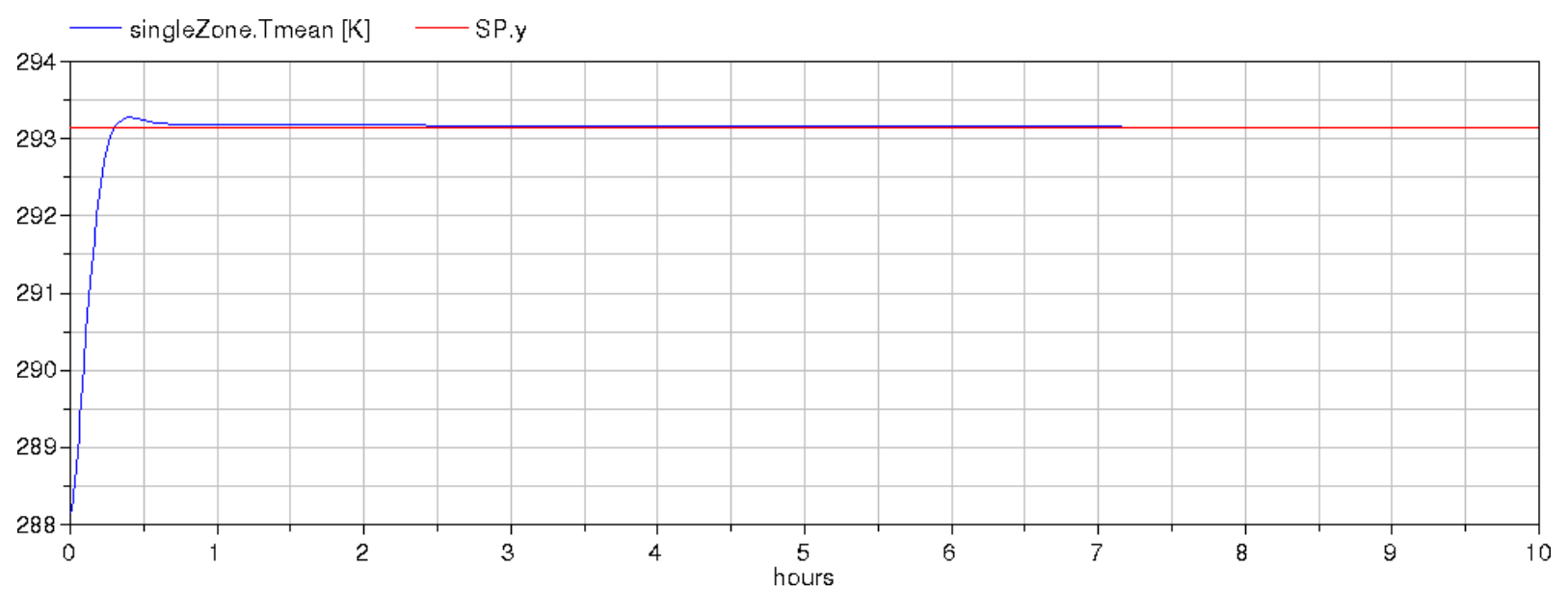

Figure 4: (Level 1) temperature of the air within the room (K).

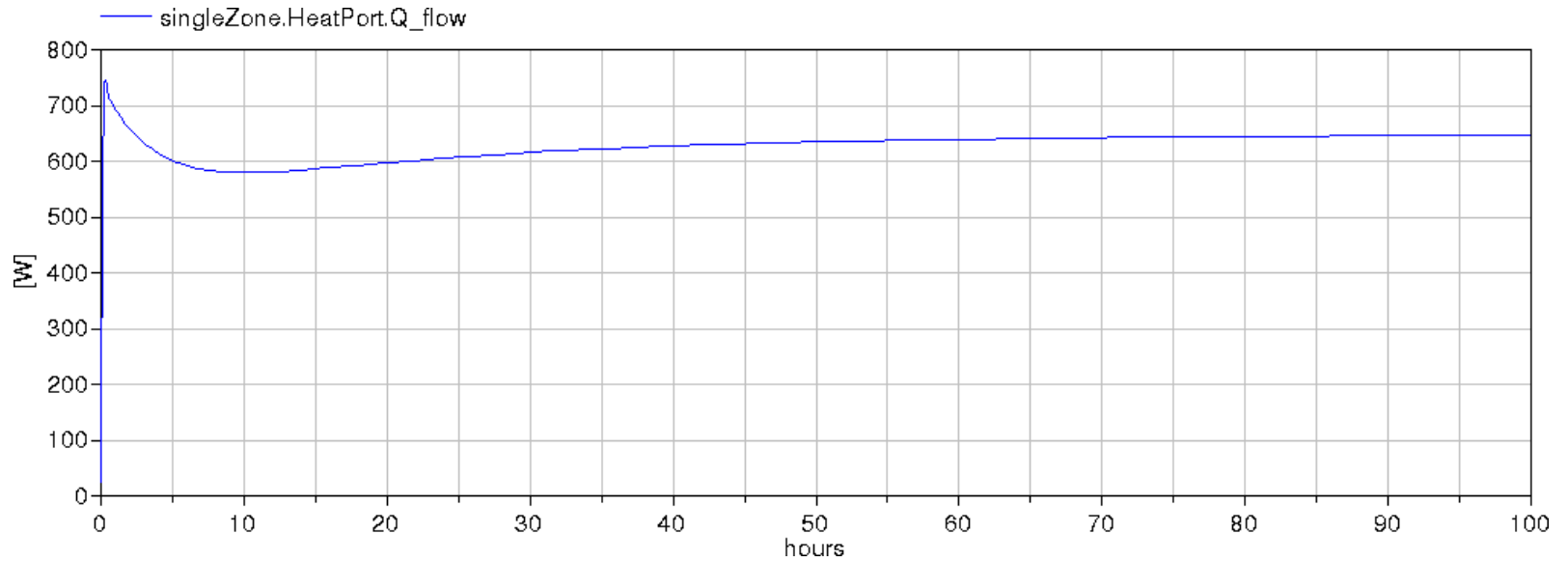

Figure 5: (Level 1) power supplied to the room (W).

the accent is posed on the heating system, that is not merely considered as an ideal heat flux injected in the room, as it was before. Given a certain quantity of hot water (assumed to be at $75^{\circ} \mathrm{C}$ ) coming into the heater, its characteristics are investigated in order to release a certain power to the air.

In figure (3), the heating system is represented by a lumped-parameter model of the heater (an exchanging tube plus a metal mass), a pump described by a head/flow characteristic, and a source and a sink node prescribing respectively the heating fluid pressure and temperature, and the discharge pressure. Connectors allow for compatibility with lower-detail models, apparently.

Of course the so obtained results need checking against the full level-2 model, which is however omitted here for brevity. Notice however how the level of detail can be scaled in a non-uniform way throughout the model: the proposed level definition is therefore just a guideline, that the flexibility of the objectoriented approach allows the analyst to tailor accord- ing to the particular question needing an answer.

\subsection{Level 3: complete model}

After sizing the main components that compose the system, the overall (level 3) model can be set up and simulated. At this level (fig. 6) both the dynamics of the room and of the heating system are taken into account, and also the central controls are represented. In fact, as can be seen, the heating system now includes a model of the boiler, accounting for the water heat balance and having as input the fuel flow rate, while the combustion process is not described and simply replaced by a fixed power released to the water and computed as the fuel flow times its heating value. Optionally a static efficiency curve can be introduced, which is however a useless detail in the context of this work.

The purpose of this analysis is the tuning of the control system. As a consequence, at this level the designer can verify that the sizing decisions previously taken are correct (and if not go back and size again). 


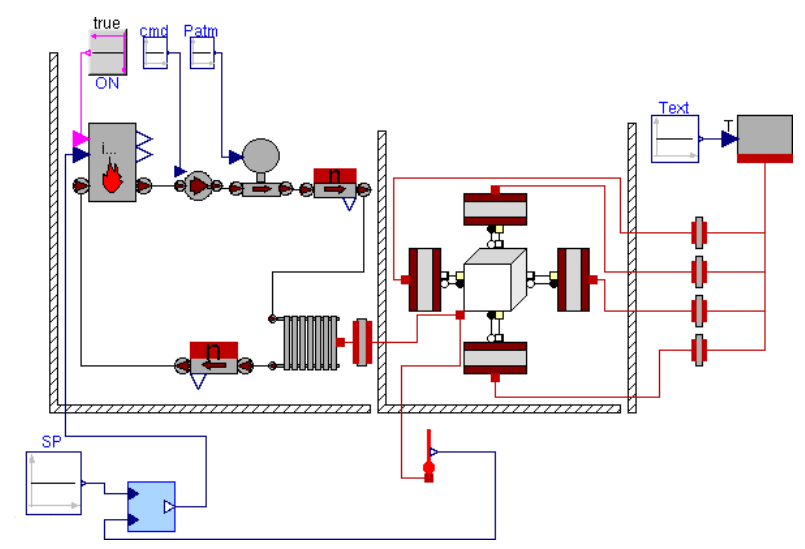

Figure 6: (Level 3) dynamic analysis of the room together with the heating system.

The tuning of the control system is done on a simple but reliable model that reflects all the dynamics that are part of the system (heating system, air and walls). The controller defined at this level may be used in more detailed descriptions.

\subsection{More detail when needed}

To further show the flexibility of the proposed modus operandi, one may need to reach even deeper levels of detail with respect to the main ones envisaged above. For example, up to now, the air within the room has always been treated as a unique entity (zero-dimensional model) and thus it had the same temperature, pressure, and so on, in every point. If necessary, the proposed model structuring allows to introduce more realistic approximations, for example based on a grid of subvolumes.

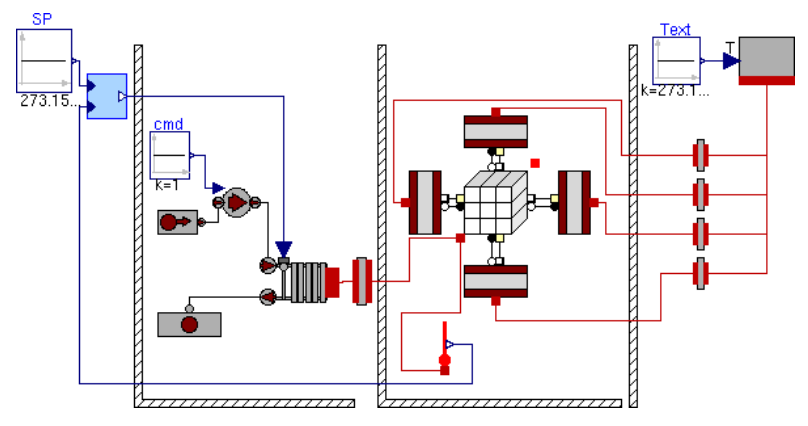

Figure 7: Dynamic analysis of the room together with the heating system. In this case the room is not considered as a single volume but is split into a coarse grid of sub-volumes.

With such a model it is possible to describe the motion of the air within the room, and more important, the temperature distribution within it. So, having a (more) detailed description of the temperature distribution of

the air contained in the room, problems like that of positioning the heater and the sensor in different places may be tackled. Notice that in figure (7) the heating system is described at an intermediate level (heater but no boiler); of course any variation is possible.

Results in figure (8) and (9) evidence how positioning the sensor in different places may vary the behaviour of the overall system. In particular, in the first case, the sensor is positioned on the left wall (the one where the heater too is placed), while in the second one, the sensor is on the opposite wall. In the first case the temperature is clearly underestimated, while in the other one it is overestimated, with the apparent consequences on transients and consumption. Simulating for 24 hours, in fact, the energy consumed in the models is $15.31 \mathrm{kWh}$ against $13.85 \mathrm{kWh}$ with a difference of $1.46 \mathrm{kWh}$. Such differences, when computing the overall consumption of a building over a year, may be significant.

On a similar front, one may want to describe in greater details (preserving the interface, of course) the energetically active components. To show an example, we report the model of the boiler in figure 6 .

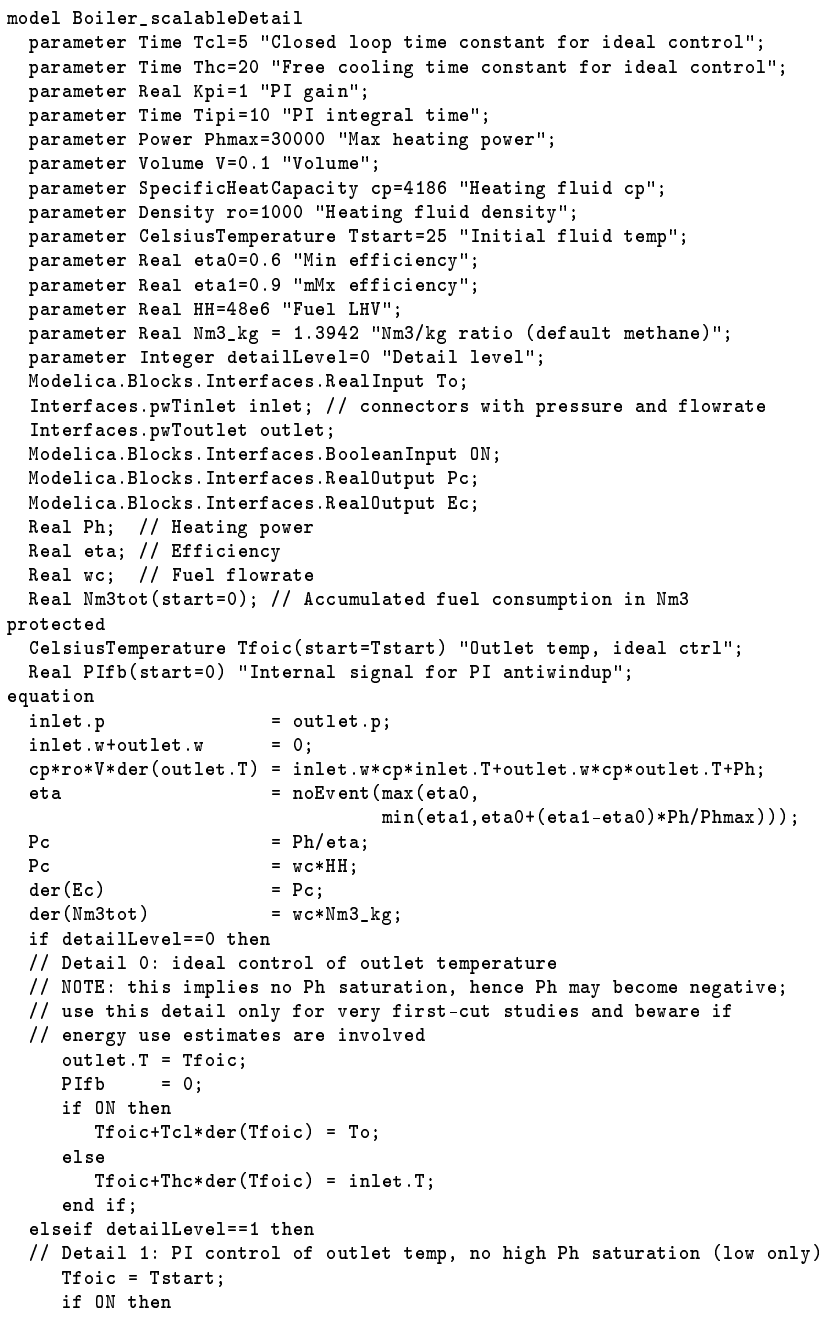




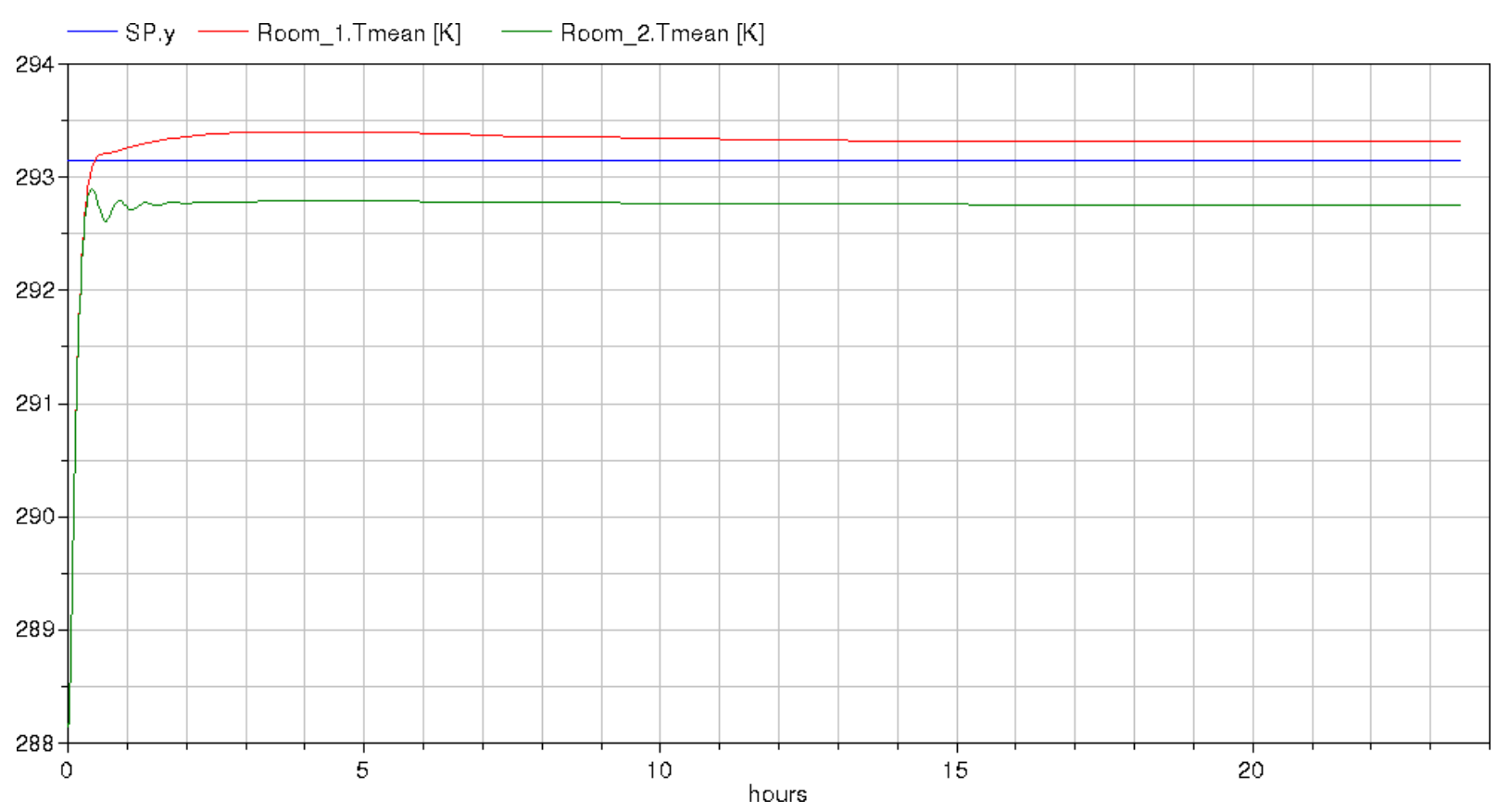

Figure 8: Measured (controlled) room temperature with different sensor positions (K).

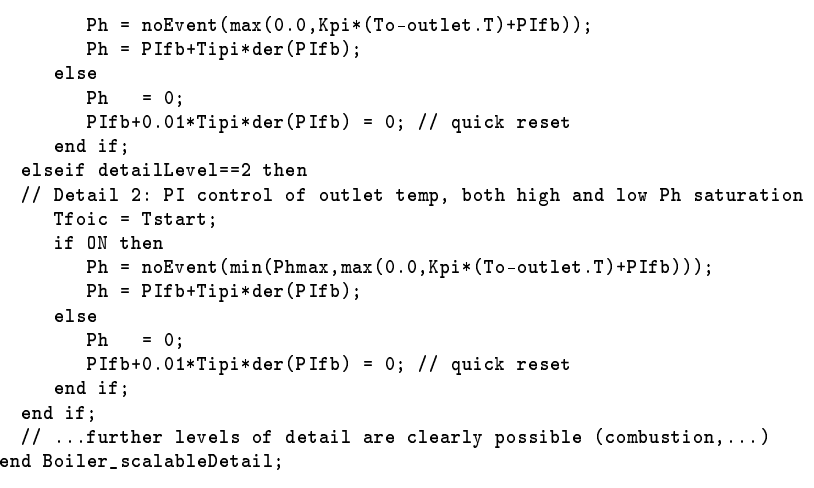

Notice how the same model can be used for sizing the equipment and assessing the central controls, consistently with the proposed way of using the simulation tool along the evolution of a project. Also, the use of convenient top-level parameters allows to use a single model, tailoring the detail level of its parts as needed. Of course the same result could have been obtained by exploiting model replaceability, but in the opinion of the authors, keeping all of a component's behaviour within a single model enhances readability (although of course the matter is largely subjective).

\section{Conclusions}

The use of object-oriented models throughout a project relative to building energy efficiency was discussed. Based on the authors' experience, one major weakness of most approaches to date is the lack of a library structuring conceived so as to follow the nec- essary modifications of the required detail level, in all or part of the model, with the maximum ease of use on the part of the designer.

Along such a reasoning, a library structuring was proposed, and preliminarily demonstrated by applying it to quite simple case, yet complete enough to be a representative example. According to such initial results, it appears that a research effort specifically aimed at an effective library structuring as perceived by the user when managing models along a project, can be very beneficial for a better acceptance of the object-oriented paradigm, and a better exploitation of its possibilities.

Ongoing research is on the realisation of a complete library based on the envisaged structuring, both integrating the available wealth of literature results, and introducing ad hoc models simplifications, especially in a view to easing the task of aligning models of different detail levels with the minimum effort.

\section{References}

[1] F. Felgner, S. Agustina, R. Caldera Bohigas, R.Merz, and L. Litz. Simulation of thermal building behaviour in Modelica. Oberpfaffenhofen, Germany, 2002.

[2] F. Felgner, R. Merz, and L. Litz. Modular modelling of thermal building behaviour using Mod- 


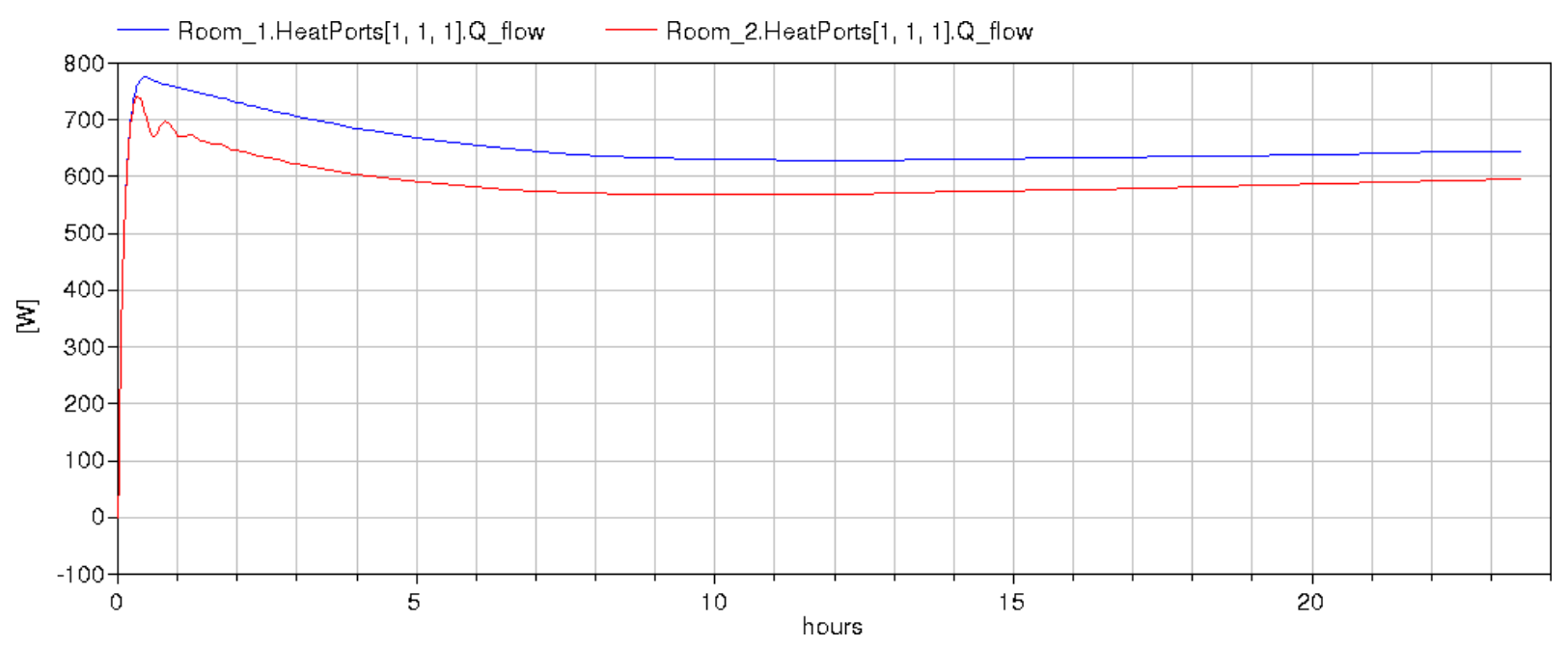

Figure 9: Power consumption with different sensor positions (W).

elica. Mathematical and computer modelling of dynamical systems, 12(1):35-49, 2006.

[3] M. Janak. Coupling building energy and lighting simulation. Kyoto, Japan, 2000.

[4] M. Wetter. Modelica-based modeling and simulation to support research and development in building energy and control systems. Journal of Building Performance Simulation, 2(1):143-161, 2009.

[5] M. Wetter. Modelica library for building heating, ventilation and air-conditioning systems. Como, Italy, 2009. 